\title{
Percepción del clima educacional en el pregrado de la carrera de cinesiología (UCSC-Chile)
}

Marcela Hechenleitner, Guillermo Flores, Verónica Bittner, Alejandra Nocetti, Andrea Meyer, Mauricio Alarcón, Claudio Lermanda

Introducción. En la actualidad hay suficiente evidencia para establecer que las variables afectivas, actitudinales y físicas influyen en el aprendizaje. Dado lo anterior, existe interés por estudiar el clima educacional y avanzar hacia el desarrollo de propuestas para su mejora. En este contexto, se desarrolló un estudio descriptivo sobre clima educacional a través de la aplicación de la encuesta Dundee Ready Educational Enviromenment (DREEM) en su versión en español, a los tres primeros años de la carrera de cinesiología de la Universidad Católica de la Santísima Concepción, Chile.

Sujetos y métodos. Se seleccionó una muestra de 142 estudiantes. Se realizó un análisis estadístico de los datos apoyados en el programa SPSS v. 19, que permitió evidenciar una alta consistencia interna medida a través del alfa de Cronbach $(0,91)$.

Resultados y conclusiones. Los resultados muestran una apreciación positiva del clima educacional, destacando la percepción sobre los profesores como factor clave. Además, se estableció que no existe relación significativa entre clima educacional y sexo o procedencia del estudiante. Finalmente, se observa una disminución de la percepción del clima educacional a medida que los alumnos avanzan en su carrera, siendo esto relevante de considerar en intervenciones necesarias en el área de la formación profesional.

Palabras clave. Cinesiología. Clima educacional. Modelo curricular basado en competencias y logro de resultados. Preclínico.

\section{Educational climate perception in undergraduate career of Kinesiology (UCSC-Chile)}

Introduction. At present there is enough evidence to establish that affective, attitudinal and physical variables influence learning. Given this, there is interest now in studying the educational climate and move towards the development of proposals for improvement. In this context, a descriptive study on educational climate was developed through the application of the Spanish version of the Dundee Ready Educational Enviromenment (DREEM) in Kinesiology students of the first three years, Universidad Católica de la Santísima Concepción, Chile.

Subjects and methods. A sample equivalent to 142 students was constructed. Evolving statistical analysis of data supported by the SPSS 19 program that show high internal consistency, measured by Cronbach's alpha (0.91).

Results and conclusions. The results show a positive appreciation of Educational Climate, emphasizing the perception of teachers as a key factor of this. It was also established that there is no significant relationship between educational climate and sex or appropriateness of the student. Finally, we observed a decrease in the perception of educational climate as students advance in their career, being considered in this relevant interventions needed in the area of professional training.

Key words. Curricular model competency based and outcomes of results. Educational climate. Kinesiology. Pre-clinical.

\section{Introducción}

El concepto de clima educacional hace referencia a las condiciones ambientales que rodean la vida de quienes se desenvuelven en instituciones académicas, así como las sensaciones que evocan las experiencias en el sistema educacional [1]. En las carreras del área de la salud, 'clima educacional' se define como el alma y espíritu del ambiente educacional y curricular [2].
El clima educacional ha sido ampliamente identificado como un factor influyente en el desarrollo de los estudiantes y su aprendizaje efectivo [3], como un poderoso predictor de satisfacción y éxito académico $[2,4,5]$.

Existen diversos instrumentos para medir el clima educacional en pregrado. Uno de ellos es la encuesta Dundee Ready Education Enviromenment (DREEM) [6], que ha sido traducida, validada y aplicada en escuelas de medicina de diferentes universidades de
Facultad de Medicina. Universidad Católica de la Santísima Concepción. Concepción, Chile.

Correspondencia: Profa. Marcela Hechenleitner Carvallo. Facultad de Medicina. Universidad Católica de la Santísima Concepción. Alonso de Ribera, 2850. Campus San Andrés. CP 403000. Concepción, Chile.

E-mail:

marcelahc@ucsc.cl

Conflicto de intereses: No declarado.

Competing interests: None declared.

(c) $2015 \mathrm{FEM}$ 
Tabla I. Interpretación de la DREEM y sus dominios (modificado de [1] para ajustar con la encuesta original [2]).

\begin{tabular}{|c|c|c|}
\hline & Puntuaciones & Interpretación \\
\hline \multirow{4}{*}{$\begin{array}{l}\text { Puntuaciones } \\
\text { totales de } \\
\text { la DREEM }\end{array}$} & $0-50$ & Muy pobre \\
\hline & $51-100$ & Con muchos problemas \\
\hline & $101-150$ & Más positivo que negativo \\
\hline & $151-200$ & Excelente \\
\hline \multirow{4}{*}{$\begin{array}{l}\text { Dominio 1: } \\
\text { percepción } \\
\text { del aprendizaje }\end{array}$} & $0-12$ & Muy pobre \\
\hline & $13-24$ & La enseñanza se percibe negativamente \\
\hline & $25-36$ & $\begin{array}{l}\text { Una percepción más bien } \\
\text { positiva de la enseñanza }\end{array}$ \\
\hline & $37-48$ & La enseñanza es muy bien evaluada \\
\hline \multirow{4}{*}{$\begin{array}{l}\text { Dominio 2: } \\
\text { percepción } \\
\text { de los docentes }\end{array}$} & $0-11$ & Abismal \\
\hline & $12-22$ & Necesitan entrenamiento educacional \\
\hline & $23-33$ & Encaminado en la dirección correcta \\
\hline & $34-48$ & La enseñanza es muy bien evaluada \\
\hline \multirow{4}{*}{$\begin{array}{l}\text { Dominio 3: } \\
\text { autopercepción }\end{array}$} & $0-8$ & Sentimientos de fracaso total \\
\hline & $9-16$ & Muchos aspectos negativos \\
\hline & $17-24$ & Sintiéndose más en el lado positivo \\
\hline & $25-32$ & Seguro del futuro académico \\
\hline \multirow{4}{*}{$\begin{array}{l}\text { Dominio 4: } \\
\text { percepción de } \\
\text { la atmósfera }\end{array}$} & $0-12$ & Un ambiente pésimo \\
\hline & $13-24$ & $\begin{array}{l}\text { Hay muchos aspectos } \\
\text { que necesitan cambiar }\end{array}$ \\
\hline & $25-36$ & Una actitud más bien positiva \\
\hline & $37-48$ & Percepción general buena \\
\hline \multirow{4}{*}{$\begin{array}{l}\text { Dominio 5: } \\
\text { percepción } \\
\text { social }\end{array}$} & $0-7$ & Miserable \\
\hline & 8-14 & No es un buen lugar \\
\hline & $15-21$ & No tan mal ambiente social \\
\hline & $22-28$ & Muy buen ambiente social \\
\hline
\end{tabular}

todo el mundo, como Brasil [7], Canadá [4], Reino Unido [8], Arabia Saudita [9], Chile [10,11], etc. Este instrumento también ha sido validado en diferentes carreras del área de la salud, como, odontología, quiropraxia, enfermería, entre otras [12-14], por lo que se concluye que es una herramienta útil para su empleo en carreras diferentes a la de medicina al entenderse que el clima educacional resulta crucial para alentar a los estudiantes a tomar más responsabilidad sobre su propio aprendizaje y para mejorar aspectos que incluyen las dimensiones físicas, sociales y académicas que el concepto conlleva.

Por otra parte, la realidad de la carrera de cinesiología de la Universidad Católica de la Santísima Concepción (UCSC) - una carrera nueva que cuenta hasta la fecha con sólo tres años y que se creó bajo un modelo formativo basado en competencias y logro de resultados- y la necesidad de verificar el desarrollo del proceso académico para garantizar su avance efectivo ha generado la exigencia de determinar el clima educacional en la carrera de cinesiología de la Facultad de Medicina de la UCSC. Para ello se ha utilizado la encuesta DREEM, que en nuestro conocimiento nunca se ha aplicado con anterioridad a estudiantes de cinesiología en Chile.

Este trabajo fue aprobado por el Consejo de Facultad de la Facultad de Medicina de la UCSC y forma parte del proyecto DIN 09/2013 de la UCSC.

\section{Sujetos y métodos}

El estudio fue de carácter descriptivo y su diseño fue de tipo transversal, midiéndose en una sola oportunidad la variable de estudio en la población en el primer semestre académico del año 2013.

La muestra correspondió a estudiantes de la carrera de cinesiología de la UCSC. Se utilizó un muestreo de tipo intencional, obteniendo una muestra de 142 participantes $(72,40$ y 30 alumnos de primer, segundo y tercer año de la carrera, respectivamente). En total se encuestó a 77 mujeres y 64 hombres (un solo alumno no respondió).

Respecto a la recogida de datos, se utilizó la DREEM, que fue obtenida en su versión en español de la validación realizada por Herrera et al [15], al igual que su escala de interpretación de resultados (Tabla I). La DREEM se compone de 50 ítems agrupados en cinco dominios: percepción del aprendizaje, percepción del docente, percepción académica, percepción de la atmósfera y percepción social.

La DREEM tiene una escala tipo Likert con cinco opciones, desde completamente de acuerdo (4 puntos) a completamente en desacuerdo (0 puntos). Los ítems $4,8,9,17,25,35,39,48$ y 50 contienen enunciados en negación, por lo que sus puntuaciones se invirtieron $[6,15]$.

La DREEM se respondió de manera anónima. El análisis de los datos se desarrolló con el programa SPSS v. 19 y correspondió fundamentalmente a estadísti- 
Tabla II. Información demográfica de la muestra ( $n=142)$.

\begin{tabular}{|c|c|c|c|}
\hline & & $n$ & $\%$ \\
\hline \multirow{2}{*}{ Sexo } & Masculino & 65 & 45,77 \\
\hline & Femenino & 77 & 54,23 \\
\hline \multirow{5}{*}{ Edad } & 16-18 años & 46 & 32,39 \\
\hline & 19-21 años & 86 & 60,56 \\
\hline & 22-24 años & 6 & 4,22 \\
\hline & 25 años o más & 1 & 0,70 \\
\hline & No responde & 2 & 1,41 \\
\hline \multirow{3}{*}{ Año académico } & Primer año & 72 & 50,70 \\
\hline & Segundo año & 40 & 28,18 \\
\hline & Tercer año & 30 & 21,13 \\
\hline \multirow{3}{*}{$\begin{array}{l}\text { Distancia entre } \\
\text { el lugar de } \\
\text { residencia y el } \\
\text { centro de estudio }\end{array}$} & Distancia < 20 km & 106 & 74,65 \\
\hline & Distancia > $20 \mathrm{~km}$ & 30 & 21,13 \\
\hline & No responde & 6 & 4,23 \\
\hline \multirow{3}{*}{$\begin{array}{l}\text { Lugar de } \\
\text { residencia }\end{array}$} & Vive en su hogar & 87 & 61,27 \\
\hline & $\begin{array}{l}\text { Vive en una pensión } \\
\text { o con familiares }\end{array}$ & 49 & 34,51 \\
\hline & No responde & 6 & 4,23 \\
\hline
\end{tabular}

ca descriptiva que permitió analizar el comportamiento de las variables en estudio a través del análisis de sus dimensiones de la escala. Además, se evaluó la fiabilidad a través de la prueba alfa de Cronbach y se trabajó con un nivel de significación del 5\%. Del mismo modo, se pusieron a prueba las hipótesis referidas a la relación entre clima educacional y variables como la distancia al centro de estudio (mayor o menor a $20 \mathrm{~km}$ ), sexo y si vive en su hogar; al ser dicotómicas estas dos últimas variables cualitativas, se codificaron con 0 y 1 . Se obtuvieron los coeficientes de regresión y el coeficiente de determinación $R^{2}$, que expresa el grado de ajuste del modelo y el poder predictivo de las variables independientes.

\section{Resultados}

La carrera de cinesiología cuenta con un total de 163 alumnos, de los cuales el 87,1\% participó en el
Tabla III. Alfa de Cronbach por dominio y año académico.

\begin{tabular}{lcccc}
\hline & 1.er. año & 2. año & 3.er. año & Total \\
\hline Percepción de la enseñanza & 0,67 & 0,63 & 0,87 & 0,74 \\
\hline Percepción del docente & 0,61 & 0,62 & 0,84 & 0,73 \\
\hline Autopercepción & 0,65 & 0,67 & 0,52 & 0,65 \\
\hline Percepción de la atmósfera & 0,67 & 0,62 & 0,77 & 0,70 \\
\hline Percepción social & 0,65 & 0,57 & 0,81 & 0,68 \\
\hline Total & 0,91 & 0,89 & 0,94 & 0,91 \\
\hline
\end{tabular}

Tabla IV. Propiedades psicométricas básicas: suma de medias totales ( \pm desviación estándar) de la encuesta DREEM.

\begin{tabular}{lcccc}
\hline & 1.er. año & 2. año & 3.er. año & Puntuación general \\
\hline Percepción de la enseñanza & $35 \pm 9,47$ & $37 \pm 8,46$ & $34 \pm 10,54$ & $35 \pm 9,62$ \\
\hline Percepción del docente & $32 \pm 8,65$ & $36 \pm 8,11$ & $36 \pm 9,11$ & $34 \pm 8,99$ \\
\hline Autopercepción & $20 \pm 10,65$ & $25 \pm 7,38$ & $24 \pm 7,84$ & $22 \pm 10,04$ \\
\hline Percepción de la atmósfera & $34 \pm 10,71$ & $31 \pm 11,40$ & $31 \pm 12,10$ & $30 \pm 11,55$ \\
\hline \begin{tabular}{l} 
Percepción social \\
\hline
\end{tabular} & $19 \pm 6,44$ & $18 \pm 6,68$ & $17 \pm 7,59$ & $18 \pm 6,93$ \\
\hline $\begin{array}{l}\text { Puntuaciones } \\
\text { generales por dominio }\end{array}$ & $139 \pm 45,93$ & $146 \pm 42,03$ & $143 \pm 47,18$ & $139 \pm 47,14$ \\
\hline
\end{tabular}

estudio $(n=142)$. La muestra tiene una media de edad de 21 años. Mayoritariamente, los alumnos viven a una distancia superior a $20 \mathrm{~km}$ del centro donde realizan sus actividades académicas. El 34,51\% de los encuestados proviene de ciudades diferentes a Concepción (Tabla II).

La consistencia interna del instrumento (DREEM) se obtuvo aplicando el alfa de Cronbach $(0,91 \%)$ (Tabla III).

La puntuación general media de la DREEM obtenida por los alumnos es de 139 (Tabla IV), que corresponde al 69,5\% de la puntuación máxima de satisfacción o percepción permitida por la DREEM (200 puntos). Esto representa una visión más positiva que negativa del clima educacional dentro de la carrera de cinesiología.

En el análisis de interpretación general, los diferentes dominios obtuvieron puntuaciones ubicadas en los dos rangos superiores de valoración según interpretación de Roff et al [6] (Tabla IV). De los cin- 
Tabla V. Resumen de resultados de la encuesta DREEM.

\begin{tabular}{|c|c|c|}
\hline & Ítem & Media \pm desviación estándar \\
\hline \multirow{13}{*}{$\begin{array}{l}\text { Percepción de } \\
\text { la enseñanza }\end{array}$} & 1. Se me estimula a participar en clases & $3,5 \pm 0,6^{a}$ \\
\hline & 7. La enseñanza es frecuentemente estimulante & $3,0 \pm 0,7$ \\
\hline & 13. La enseñanza se centra en el estudiante & $3,2 \pm 0,7$ \\
\hline & 16. La enseñanza me ayuda a desarrollar mis competencias & $3,3 \pm 0,7$ \\
\hline & 20. La enseñanza está bien enfocada & $3,2 \pm 0,7$ \\
\hline & 21. Siento que me están preparado bien para mi profesión & $3,5 \pm 0,8^{a}$ \\
\hline & 24. El tiempo destinado a la enseñanza es bien utilizado & $3,1 \pm 0,8$ \\
\hline & 25. La enseñanza de la carrera pone demasiado énfasis en el aprendizaje de detalles & $1,3 \pm 1,0^{b}$ \\
\hline & 38. Tengo claros los objetivos de aprendizaje de mis cursos & $2,9 \pm 0,9$ \\
\hline & 44. La manera de enseñar me estimula a aprender por mí mismo de forma activa & $2,9 \pm 0,8$ \\
\hline & 47. En la carrera se enfatiza el aprendizaje a largo plazo sobre el inmediato & $2,7 \pm 1,0$ \\
\hline & 48. La enseñanza de la carrera está demasiado centrada en el docente & $2,6 \pm 1,0$ \\
\hline & Total por dominio & $2,9 \pm 0,6$ \\
\hline \multirow{12}{*}{$\begin{array}{l}\text { Percepción } \\
\text { del docente }\end{array}$} & 2. Los docentes conocen la materia que dictan & $3,7 \pm 0,5^{a}$ \\
\hline & 6. Los docentes clínicos tienen paciencia con los pacientes & $2,7 \pm 0,9$ \\
\hline & 8. Los docentes ridiculizan a los estudiantes & $3,2 \pm 0,9$ \\
\hline & 9. Los docentes son autoritarios & $2,3 \pm 1,2$ \\
\hline & 18. Los docentes tienen buenas destrezas comunicativas con los pacientes & $2,6 \pm 0,8$ \\
\hline & 29. Los docentes son buenos retroalimentando a los estudiantes & $3,2 \pm 0,7$ \\
\hline & 32. En la carrera, los docentes nos hacen críticas constructivas & $3,3 \pm 0,6$ \\
\hline & 37. Los docentes dan ejemplos claros & $3,2 \pm 0,6$ \\
\hline & 39. Los docentes se molestan y alteran las clases & $2,8 \pm 1,1$ \\
\hline & 40. Los docentes están bien preparados para sus clases & $3,4 \pm 0,6$ \\
\hline & 49. Siento que puedo hacer todas las preguntas que quiero & $3,2 \pm 1,0$ \\
\hline & Total por dominio & $3,1 \pm 0,4$ \\
\hline \multirow{9}{*}{ Autopercepción } & 5. Los métodos de estudio que tenía antes todavía me sirven & $2,2 \pm 1,2$ \\
\hline & 10. Tengo confianza de que voy a aprobar este año & $3,3 \pm 0,8$ \\
\hline & 22. La enseñanza de la carrera se preocupa suficientemente de desarrollar mi confianza & $3,2 \pm 0,8$ \\
\hline & 26. Lo aprendido el año pasado fue una buena base para el trabajo de este año & $2,7 \pm 0,9$ \\
\hline & 27. Soy capaz de memorizar todo lo que me es necesario & $2,2 \pm 1,0$ \\
\hline & 31. He aprendido mucho sobre la empatía de mí profesión & $3,2 \pm 0,9$ \\
\hline & 41. La carrera me ayuda a desarrollar mis destrezas para resolver problemas & $3,3 \pm 0,7$ \\
\hline & 45. Mucho de lo que aprender me parece relevante para la carrera profesional & $3,4 \pm 0,7$ \\
\hline & Total por dominio & $2,9 \pm 0,5$ \\
\hline
\end{tabular}


Tabla V. Resumen de resultados de la encuesta DREEM (cont.).

\begin{tabular}{|c|c|c|}
\hline & Ítem & Media \pm desviación estándar \\
\hline \multirow{13}{*}{$\begin{array}{l}\text { Percepción de } \\
\text { la atmósfera }\end{array}$} & 11. El ambiente es relajado durante las visitas docentes de los servicios hospitalarios & $2,4 \pm 0,6$ \\
\hline & 12. Los horario de la carrera están bien programados & $1,6 \pm 1,2^{b}$ \\
\hline & 17. En la carrera, la copia en los exámenes constituye un problema & $0,9 \pm 1,0^{b}$ \\
\hline & 23. El ambiente es relajado durante las clases teóricas en el aula & $2,8 \pm 0,8$ \\
\hline & 30. Tengo oportunidades para desarrollar mis habilidades interpersonales & $2,9 \pm 0,8$ \\
\hline & 33. Me siento cómodo, socialmente, en las clases & $3,1 \pm 1,0$ \\
\hline & 34. El ambiente en los seminario, clases y prácticas es relajado & $2,7 \pm 0,9$ \\
\hline & 35. Mi experiencia en la carrera ha sido desalentadora & $3,1 \pm 1,0$ \\
\hline & 36. Soy capaz de concentrarme bien & $2,6 \pm 1,0$ \\
\hline & 42. Disfrutar de mis estudios en la carrera pesa más que la tensión que éstos me generan & $2,3 \pm 1,2$ \\
\hline & 43. El ambiente de la carrera me motiva para aprender & $3,1 \pm 0,8$ \\
\hline & 50. Los estudiantes causamos irritación a los docentes & $2,5 \pm 1,1$ \\
\hline & Total por dominio & $2,5 \pm 0,7$ \\
\hline \multirow{8}{*}{$\begin{array}{l}\text { Percepción } \\
\text { social }\end{array}$} & 3. Hay un buen sistema de apoyo para los estudiantes que sufren estrés & $2,0 \pm 0,8$ \\
\hline & 4. Estoy demasiado cansado para disfrutar de los cursos que estoy siguiendo & $2,0 \pm 1,1$ \\
\hline & 14. Rara vez me aburro en los cursos que estoy siguiendo & $2,5 \pm 1,1$ \\
\hline & 15. Tengo buenos amigos en la carrera & $3,2 \pm 0,9$ \\
\hline & 19. Mi vida social es buena & $3,1 \pm 0,9$ \\
\hline & 28. Rara vez me siento solo & $2,7 \pm 1,1$ \\
\hline & 46. Los ambientes físicos de la facultad son agradables & $2,8 \pm 1,1$ \\
\hline & Total por dominio & $2,6 \pm 0,5$ \\
\hline \multicolumn{2}{|c|}{ Promedio general } & $2,8 \pm 0,3$ \\
\hline
\end{tabular}

Algunos términos fueron cambiados para ajustarse mejor a la realidad de la carrera, sin modificar el sentido de la sentencia. a ítems realmente positivos (promedio de 3,5); b ítems negativos en los cuales debe intervenirse (promedio $<2,0$ ).

co dominios que comprende la DREEM, la percepción del docente alcanzó la más alta interpretación, correspondiente a 'docentes modelos' (Tabla IV). Los otros cuatro dominios (percepción de la enseñanza, autopercepción, percepción de la atmósfera y percepción social) tienen una interpretación positiva, alcanzando el segundo rango de interpretación de la DREEM (Tabla IV). El alfa de Cronbach para cada dominio confirma la consistencia interna de la DREEM (Tabla IV).

El clima educacional en los cinco dominios presenta una media de respuestas entre 2,5 y 3,1 (Tabla V). Los ítems 1 y 21 del dominio de percepción de la enseñanza obtuvieron una media de respuesta sobre 3,5, al igual que el ítem 2 del dominio de percepción docente $(3,7)$, lo que equivale a un $6 \%$ de ítems positivos con excelente percepción (Tabla V). El 86\% de los ítems obtuvieron una media de respuesta de 2,8 , lo que indica que pueden enriquecerse (Tabla V). El 6\% califica como áreas con problemas aquellos ítems con una media de respuestas de 1,3 (ítem 25, 12 y 17) (Tabla V).

$\mathrm{Al}$ analizar los años por separado se observaron puntuaciones generales medias de 139, 146 y 143 para primer, segundo y tercer año, respectivamente (Tabla IV), lo que corrobora el análisis de regresión 
Tabla VI. Regresión lineal múltiple entre percepción del clima educacional y las variables explicativas para la carrera de cinesiología.

\begin{tabular}{lccc}
\hline & Coeficiente $\beta$ & $\begin{array}{c}\text { IC 95\% para } \\
\text { el coeficiente } \beta\end{array}$ & $p$ \\
\hline Constante & 2,5527 & $2,4052-2,7003$ & 0,0000 \\
\hline Año de estudio & 0,1456 & $0,0664-0,2249$ & 0,0004 \\
\hline
\end{tabular}

$R^{2}=9,22 \%$. IC 95\%: intervalo de confianza al 95\%. Variables excluidas: sexo, distancia al hogar, vive en su hogar.

$\left(p=0,0004 ; R^{2}=9,22 \%\right)$ (Tabla VI). El resto del análisis de regresión de los datos muestra que no hay diferencias significativas entre la percepción del clima educacional y las variables sexo, lejanía entre residencia y el centro de estudio (distancia del centro de estudio) y si el estudiante vive o no con su familia (Tabla VI).

\section{Discusión}

La DREEM tuvo una alta tasa de respuesta $(87,1 \%)$, lo que indica una alta representatividad de los resultados. Los resultados generales muestran un clima educacional calificado como más positivo que negativo, ubicado en la parte media para el tramo definido para dicha denominación según Roff et al [6] y la versión en español de Herrera et al [15]. Si bien estamos frente a una percepción positiva, existen algunas áreas que deben mejorarse para alcanzar el tramo superior correspondiente a una percepción de excelencia del clima educacional.

La percepción de la enseñanza (dominio 1) se observa en general con una visión positiva. Los dos primeros años de la carrera evalúan muy bien este dominio y en el tercer año la percepción disminuye levemente, aunque siempre se considera positiva. La percepción de los docentes (dominio 2) permanece constante en los dos últimos años evaluados de carrera como 'modelo'. La autopercepción (dominio 3) es un poco superior en los dos primeros años de carrera, y en general se percibe de manera positiva. La atmósfera (dominio 4) y la percepción social (dominio 5) se evalúan como positivas, aunque no están en el rango superior según la interpretación de Roff et al [6]. Estos resultados indican que debemos realizar ajustes para alcanzar la excelencia en este tema y así mejorar el clima educacional, lo que conlleva una mejora en el rendimiento académico de los alumnos $[2,4,5]$.
Los bajos porcentajes que se observan frente a los aspectos clínicos (ítems 6 y 11) pueden estar dados por la poca participación en actividades clínicas con pacientes reales en los primeros años de la carrera. Hasta el momento no sabemos si esta área sufre cambios al avanzar en su itinerario formativo, ya que aún no contamos con promociones que abarquen la carrera en su totalidad. Las prácticas clínicas en el itinerario formativo aumentan progresivamente hacia los últimos años de la carrera, siendo la actividad principal durante su residencia, lo que lleva a conjeturar que la percepción positiva de esta área debería ir mejorando.

Para mejorar el clima educacional percibido en la carrera de cinesiología se hace necesario realizar intervenciones que corrijan los ítems relacionados con el detalle de los contenidos (ítem 25), la relación entre estilo de aprendizaje y metodologías utilizadas (ítem 5), los horarios (ítem 12), la supervisión de exámenes (ítem 17) y los sistemas de apoyo al estudiante (ítems 3 y 4 ) respecto de conductas de afrontamiento frente al estrés generado por las exigencias propias del ambiente universitario.

Las variables sexo, distancia del centro de estudio y si vive o no con sus padres no son predictivamente significativas de la percepción del clima educacional en la carrera de cinesiología de la UCSC $(p>0,05)$. Los datos muestran que solamente el año de estudio en que se encuentra el alumno se relaciona significativamente con su percepción del clima educacional (mejor percepción en los primeros años; $p=0,0004$ ). El coeficiente de determinación $\left(R^{2}=9,22 \%\right)$ indica que la variabilidad en la percepción que tiene el alumno sobre el clima educacional puede explicarse en un 9,225 por el año de estudio en que se encuentra.

La carrera de cinesiología (con sólo tres años) obedece a un modelo basado en competencias y logros de resultados, y desde el punto de vista del clima educacional podemos concluir que se encuentra bien encaminada, lo que no significa que no se pueda mejorar. Lamentablemente no tenemos referencias bibliográficas para comparar nuestros resultados con otras carreras de cinesiología en Chile, pero si realizamos una comparación con los estudios de DREEM que se han hecho en Chile en las carreras de medicina [15], podemos ver que nuestra carrera presenta una percepción del clima educacional considerablemente superior a la encontrada en seis universidades chilenas, incluida la UCSC.

Este trabajo sugiere que la encuesta DREEM es una herramienta válida para medir el clima educacional en la carrera de cinesiología. La información recogida a través de la encuesta DREEM puede ser 
útil para las autoridades de la carrera de cinesiología en el programa de desarrollo profesional. Nosotros proponemos estudios adicionales en diferentes escuelas de cinesiología nacionales y recomendamos aumentar el tamaño de la muestra para validar la estabilidad y estructura factorial de la encuesta DREEM.

Bibliografía

1. Chassin-Trubert I, Durán J. Percepción de los estudiantes del Instituto Virginio Gómez sobre el clima educacional. Rev Educ Cienc Salud 2001; 8: 9-14.

2. Genn JM. AMEE Medical Education Guide No. 23 (Part 2). Curriculum, environment, climate, quality and change in medical education -a unifying perspective. Med Teach 2001; 23: 445-54.

3. Seabrook M. Clinical students' initial reports of the educational climate in a single medical school. Med Educ 2004; 38: 659-69.

4. Till H. Identifying the perceived weaknesses of a new curriculum by means of the Dundee Ready Education Environment Measure (DREEM) Inventory. Med Teach 2004; 26: 39-45.

5. Lizzio A, Wilson K, Simons R. University students' perceptions of the learning environment and academic outcomes: implications for theory and practice. Studies Higher Educ 2002; 27: 27-52.

6. Roff S, Mcaleer S, Harden RM, Al-Qahtani M, Uddin AA, Deza H, et al. Development and validation of the Dundee
Ready Education Environment Measure (DREEM). Med Teach 1997; 19: 295-9.

7. Sobral DT. Medical students' self-appraisal of first-year learning outcomes: use of the course valuing inventory. Med Teach 2004; 26: 234-8.

8. Dunne FS, McAleer S, Roff S. Assessment of the undergraduate medical education environment in a large UK medical school. Health Educ J 2006; 65: 149-58.

9. Hasan T, Gupta P. Assessing the learning environment at Jazan medical school of Saudi Arabia. Med Teach 2013; 35: S90-6.

10. Riquelme A, Oporto J, Méndez J, Viviani P, Salech F. Measuring students' perceptions of the educational climate of the new curriculum at the Pontificia Universidad Católica de Chile: performance of the Spanish translation of the Dundee Ready Education Environment Measure (DREEM). Educ Health 2009; 22: 1 .

11. Valdebenito M, Rivera A, Kirsten L. Percepción de los estudiantes de medicina sobre el clima educacional en las áreas preclínica y clínica. Rev Educ Cienc Salud 2008; 4: 147.

12. Said NM, Rogayah J, Hafizah A. A study of learning environment in the Kulliyyah (Faculty) of Nursing, International Islamic University Malaysia. Malaysian J Med Sci 2009; 16: 11-20.

13. Palmgren P. Perception of educational environment among undergraduate students in a chiropractic training institution. J Chiropract Educ 2011; 25: 151-63.

14. Soemantri D, Herrera, C, Riquelme, A. Measuring the educational environment in health professions studies: a systematic review. Med Teach 2010; 32: 947-52.

15. Herrera C, Pacheco J, Rosso F, Cisterna C, Aichelle D, Becker S, et al. Evaluación del ambiente educacional preclínico en seis escuelas de medicina en Chile. Rev Med Chile 2010; 138: 677-84. 